\title{
The Number of Periodic Solutions of Some Analytic Equations of Abel Type*
}

\author{
Naeem Alkoumi ${ }^{\dagger}$ \\ Departamento de Matemática Aplicada, \\ Universidad de Granada, 18071 Granada, Spain.
}

\begin{abstract}
New result are proved to estimate the number of periodic solutions of a differential equation of Abel type by using a modification of a technique introduced by Ilyashenko. The main tool is an estimate on the number of zeros of a holomorphic function. A concrete example is analyzed but the results are presented to make the method flexible and applicable to other equations.
\end{abstract}

Keywords: Abel-type equation, periodic solution, holomorphic function, Christoffel-Schwarz formula.

\section{Introduction}

In [1] Ilyashenko considered the differential equation of polynomial type

$$
x^{\prime}=x^{n}+\sum_{j=0}^{n-1} a_{j}(t) x^{j}
$$

with $a_{j}: \mathbb{R} \longrightarrow \mathbb{R}$ continuous and 1-periodic. Assuming that all the coefficients $a_{j}(t)$ were dominated by a common bound $C \geq 1$, he obtained an estimate (depending on $n$ and $C$ ) on the number of periodic solutions of equation (1). The purpose of this paper is to show that this method can also be applied to more general equations. As a model we will consider the equation

$$
x^{\prime}=x^{3}+\sin x+p(t), \quad p(t+1)=p(t)
$$

and we will obtain an estimate on the number of periodic solutions depending only on

$$
\|p\|_{\infty}=\max _{t \in[0,1]}|p(t)| \leq C .
$$

*Supported by project FQM2216, Junta de Andalucía.

†n26eem@ugr.es 
The basic tool employed in [1] is the so called Jensen's Lemma. This is a result in Complex Analysis that allows to estimate the number of zeros of a holomorphic function in a domain $D$ in terms of the behavior on the boundary $\partial D$. The standard version of this Lemma assumes that $D$ is a disc but the result can be transported to other domains via Riemann's Theorem on conformal mappings. The approach in [1] was to consider certain domains with the shape of a stadium and to employ ideas taken from hyperbolic geometry to estimate some quantities related to the Riemann's mapping for these domains. Our approach will be more straightforward, we will consider the explicit Christoffel-Schwarz formula mapping the unit disc onto a rectangle. This will allow us to state a version of Jensen's Lemma for the rectangle where all the quantities involved are explicit and expressed in terms of elliptic functions. Once we have obtained this result in Complex Analysis we will present a modified version of Ilyashenko's technique applicable to equation (2). It seems to us that the results are presented so that the method is flexible and can be applied to many other equations.

\section{The number of zeros of an analytic function}

Let $F$ be a closed rectangle with vertices $\pm a \pm i b$ and consider $h: F \rightarrow \mathbb{C}$ holomorphic in the interior of $F$ and continuous on $F$. Assume also that

$$
\overline{h(z)}=h(\bar{z}) \quad \forall z \in F,
$$

and so $h$ maps the interval $[-a, a]$ into $\mathbb{R}$.

Given any subset $A$ of $F$ we employ the notation $N(h, A)$ to indicate the number of zeros of $h$ on $A$. Zeros are counted according to their multiplicity. Assume that we are given a real interval $J=[-\Gamma, \Gamma]$ with $\Gamma<a$, we would like to find an estimate on $N(h, J)$.

To state our result we need the Christoffel-Schwarz transformation $f$ mapping the unit disk $D=\{z \in \mathbb{C}:|z|<1\}$ conformally onto $F$ and such that $f(0)=0$ and $f^{\prime}(0)>0$. Following [5] such a map can be expressed as

$$
f(z)=\frac{2 b}{K(\sin \alpha)} \int_{0}^{z} \frac{d \xi}{\sqrt{1-2 \cos (2 \alpha) \xi^{2}+\xi^{4}}}, \quad \frac{b}{a}=\frac{K(\sin \alpha)}{K(\cos \alpha)},
$$

where $K$ is the complete elliptic integral of the first kind.

Theorem 1 Let $J=[-\Gamma, \Gamma]$ and $h: F \rightarrow \mathbb{C}$ be in the above conditions. In addition assume that for some positive numbers $m$ and $M$,

$$
|h| \leq M \quad \text { in } \quad F, \quad \max _{J}|h|=m .
$$

Then

$$
N(h, J) \leq-\ln \left(m^{-1} M\right) / \ln \left(\frac{2 \gamma}{1+\gamma^{2}}\right),
$$

where $\gamma$ is such that $f(\gamma)=\Gamma$. 


\section{Example 1 (Test example):}

Consider the function $h(z)=\sin z$, the interval $J=\left[-\frac{\pi}{6}, \frac{\pi}{6}\right]$ and the rectangle $F=[-\pi, \pi] \times[-1.5,1.5]$. From $\frac{3}{2 \pi}=\frac{K(\sin \alpha)}{K(\cos \alpha)}$ we deduce that $\alpha=0.180762$ and $\gamma=0.287904$. The estimate $|\sin z| \leq \cosh (\Im m z)$ implies that we can take $M=\cosh (1.5)$. Since $m=0.5$ we obtain

$$
N(h, J) \leq-\frac{1}{\ln \left(\frac{2 \gamma}{1+\gamma^{2}}\right)} \ln (2 \cosh (1.5)) \approx 2.4518 .
$$

An alternative approach would consist in applying Corollary 1 of [1]. In this case the rectangle has to be replaced by a domain with the shape of a stadium. In the notations of [1] we take $K=\left[-\frac{\pi}{6}, \frac{\pi}{6}\right]$ and $U$ the $\epsilon$ - neighborhood of $K$ with $\epsilon=1.5$. Notice that $U \subset F$. We notice that $|K| \epsilon^{-1}=\frac{\pi}{3} \cdot \frac{2}{3}>\ln 2$ and so the result in [1] is applicable. The estimate on the number of zeros now become

$$
N(h, J) \leq e^{\frac{2 \pi}{9}} \ln (2 \cosh (1.5)) \approx 3.11265 .
$$

The proof will consist in an adaptation of the classical Jensen's Lemma (usually stated on a disk) to the rectangle $F$. We first recall this result.(see [2] and $[4])$.

Lemma 1 (Jensen's Lemma) Let $D=\{z \in \mathbb{C}:|z|<1\}$ be the unit disk and let $H: D \subset \mathbb{C} \rightarrow \mathbb{C}$ be a holomorphic function. For each $\rho \in(0,1)$ such that $H(z) \neq 0$ if $|z|=\rho$, we denote by $N_{\rho}$ the number of zeros (counting multiplicities) of $H$ in $|z|<\rho$. Then

$$
N_{\rho} \leq-\frac{1}{\ln \rho} \ln \frac{M}{|H(0)|},
$$

where $M=\sup _{z \in D}|H(z)|$.

In the proof we will also employ the Mobius transformation

$$
\mathcal{M}: w=\frac{z_{0}-z}{1-\overline{z_{0}} z},
$$

where $z_{0}$ is a given point satisfying $\left|z_{0}\right|<1$. This transformation maps the unit disk $D$ onto itself and it is an involution; that is, the inverse $\mathcal{M}^{-1}=\mathcal{M}$. Moreover $\mathcal{M}(0)=z_{0}$.

Proof of theorem 1. From the Christoffel-Schwarz formula we observe that if $z \in D, f(-z)=-f(z)$. Moreover $f(z) \in \mathbb{R}$ whenever $z \in \mathbb{R}$. Then $f([-\gamma, \gamma])=$ $J$ and we can pick points $\xi_{0} \in J$ and $z_{0} \in[-\gamma, \gamma]$ satisfying

$$
\left|h\left(\xi_{0}\right)\right|=m, \quad f\left(z_{0}\right)=\xi_{0} .
$$

Since $z_{0}$ is real the Mobius transformation $\mathcal{M}$ maps the real line onto itself and one immediately checks that

$$
\mathcal{M}([-\gamma, \gamma])=\mathcal{M}^{-1}([-\gamma, \gamma])=\left[\frac{z_{0}-\gamma}{1-z_{0} \gamma}, \frac{z_{0}+\gamma}{1+z_{0} \gamma}\right] .
$$


The function $\varphi(x)=\frac{\gamma+x}{1+\gamma x}, x \in[-\gamma, \gamma]$ is increasing and so

$$
\max \left\{\left|\frac{z_{0}+\gamma}{1+z_{0} \gamma}\right|,\left|\frac{z_{0}-\gamma}{1-z_{0} \gamma}\right|\right\} \leq \frac{2 \gamma}{1+\gamma^{2}}:=R<1 .
$$

These computations imply that hence $\mathcal{M}^{-1}([-\gamma, \gamma])$ is contained in the disk $|z| \leq R$.

Next we define $H=h \circ f \circ \mathcal{M}$, and observe that $|H(0)|=m$ and $|H| \leq M$ on $D$. Since $H(0) \neq 0$ we can apply the Identity Principle to find a sequence of numbers $\rho_{n} \searrow R$ and such that $H(z) \neq 0$ if $|z|=\rho_{n}$. From Jensen's Lemma applied to $H$ we deduce that

$$
N_{\rho_{n}} \leq-\frac{1}{\ln \left(\rho_{n}\right)} \ln \left(\frac{M}{m}\right)
$$

Letting $n \rightarrow \infty$ we deduce that

$$
N(h, J) \leq \lim _{n \rightarrow \infty} N_{\rho_{n}} \leq-\frac{1}{\ln (R)} \ln \left(\frac{M}{m}\right) .
$$

Assume now that $\epsilon>0$ is a fixed number and $\Gamma$ is a parameter satisfying

$$
0<\Gamma \leq \bar{\Gamma}<\infty,
$$

for a fixed $\bar{\Gamma}$. Consider the rectangle $F_{\Gamma}=[-\Gamma-\epsilon, \Gamma+\epsilon] \times[-\epsilon, \epsilon]$ and the interval $J_{\Gamma}=[-\Gamma, \Gamma]$.

Theorem 2 There exists $\vartheta=\vartheta(\bar{\Gamma}, \epsilon)>0$ such that for each $\Gamma$ satisfying (4) and each continuous function $h: F_{\Gamma} \rightarrow \mathbb{C}$ holomorphic in the interior of $F_{\Gamma}$,

$$
N\left(h, J_{\Gamma}\right) \leq \vartheta \ln \left(m^{-1} M\right),
$$

where $|h| \leq M$ in $F_{\Gamma}$ and $\max _{J_{\Gamma}}|h| \geq m$.

Remark 1 The above result is also applicable to a non-symmetric interval. Assuming $J=[a, b]$ with $0<\frac{b-a}{2} \leq \bar{\Gamma}$, we define $c=\frac{a+b}{2}$ and apply Theorem 2 to $h_{1}(z)=h(z+c)$ with $J_{c}=[-c, c], F_{c}=[-c-\epsilon, c+\epsilon] \times[-\epsilon, \epsilon]$.

Proof. The function $K(k)=\int_{0}^{\pi / 2} \frac{d \theta}{\sqrt{1-k^{2} \sin ^{2} \theta}}$ is strictly increasing on $k \in[0,1[$. In consequence also the function

$$
\alpha \in\left[0, \pi / 2\left[\mapsto \frac{K(\sin \alpha)}{K(\cos \alpha)} \in[0, \infty[\right.\right.
$$

is strictly increasing. Given $\Gamma \geq 0$ there exist a unique $\alpha=\alpha(\Gamma)$ with

$$
\frac{\epsilon}{\Gamma+\epsilon}=\frac{K(\sin \alpha)}{K(\cos \alpha)},
$$


and the function $\Gamma \in[0, \infty[\mapsto \alpha(\Gamma) \in] 0, \pi / 4]$ is strictly decreasing and continuous. Consider the Christoffel-Schwarz transformation depending upon the parameter $\Gamma$,

$$
f(z, \Gamma)=\frac{2 \epsilon}{K(\sin \alpha(\Gamma))} \int_{0}^{z} \frac{d \xi}{\sqrt{1-2 \cos (2 \alpha(\Gamma)) \xi^{2}+\xi^{4}}}
$$

where we restrict $z$ to the real axis we observe that the function $z \in[-1,1] \mapsto$ $f(z, \Gamma)$ is strictly increasing. Since $f(0, \Gamma)=0$ and $f(1, \Gamma)=\Gamma+\epsilon$ we find a unique $\gamma=\gamma(\Gamma) \in[0,1[$ with

$$
f(\gamma, \Gamma)=\Gamma
$$

Moreover $\gamma=\gamma(\Gamma)$ is a continuous function and we can define

$$
\gamma_{*}=\max _{[0, \bar{\Gamma}]} \gamma(\Gamma)
$$

We can apply Theorem 1 in $F_{\Gamma}$ and define

$$
\vartheta=-\frac{1}{\ln \left(\frac{2 \gamma_{*}}{1+\gamma_{*}^{2}}\right)} .
$$

Remark $2 \vartheta$ can be computed numerically using (5), (7) and (8).

\section{Estimates for real-valued solutions}

Consider the differential equation

$$
x^{\prime}=v(x, t)
$$

where $v: \mathbb{R} \times \mathbb{R} \rightarrow \mathbb{R}$ is continuous and 1-periodic in $t$. We also assume that there is uniqueness for the initial value problem. Given $\xi \in \mathbb{R}$ the solution satisfying $x(0)=\xi$ will be denoted by $x(t, \xi)$ and the corresponding maximal interval $] \alpha, \omega[$ with $-\infty \leq \alpha=\alpha(\xi)<0<\omega=\omega(\xi) \leq+\infty$.

Let $\mathcal{D}$ be the set of initial conditions such that the solution is well defined in $[0,1]$; that is,

$$
\mathcal{D}=\{\xi \in \mathbb{R}: \omega(\xi)>1\} .
$$

We recall that $\mathcal{D}$ in an open interval which sometimes can be empty. For instance, this is the case for $v(x, t)=\lambda\left(1+x^{2}\right)$ with $\lambda \geq \pi$.

The Poincare map is defined as

$$
P: \mathcal{D} \rightarrow \mathbb{R}, \quad P(\xi)=x(1, \xi) .
$$

This is an increasing and continuous function and periodic solutions do correspond to fixed points of $P$. 
Lemma 2 Assume that for some $r \in \mathbb{R}$

$$
v(x, t)>0 \quad \text { if } \quad x \geq r .
$$

In addition there exists a periodic solution and another solution that blows up not later than $t=1$; that is, $\omega(\xi) \leq 1$ for some $\xi \in \mathbb{R}$. Then

$$
P(\mathcal{D})=] B,+\infty[\text { for some } B, \quad-\infty \leq B<\infty .
$$

Proof. Let $A$ be an initial condition such that $x(t, A)$ is periodic solution of (9). Then $A=P(A) \in P(\mathcal{D})$. Since $P(\mathcal{D})$ is an open interval we can express it as $P(\mathcal{D})=] B, C$ [ with $-\infty \leq B<A \leq C \leq+\infty$. It remains to prove that $C=+\infty$. To this end we take any number $y \in] A,+\infty[$ and consider the solution $\phi(t)$ of (9) satisfying $\phi(1)=y$. We claim that $\phi(t)$ is well defined in $[0,1]$. Otherwise there should exist some $\alpha \in[0,1[$ such that one of the following alternatives holds,

$$
\text { (i) } \lim _{t \searrow \alpha} \phi(t)=-\infty \quad(i i) \lim _{t \searrow \alpha} \phi(t)=+\infty .
$$

The first alternative is ruled out because, by uniqueness, $\phi(t)>x(t, A)$ if $t \in$ ]$\alpha, 1]$. Assume by a contradiction argument that (ii) holds. Then, for $t$ close enough to $\alpha$ one should have $\phi(t)>r$. In consequence $\phi^{\prime}(t)=v(\phi(t), t)>0$ and $\phi$ is strictly increasing on some interval of the type $] \alpha, \alpha+\delta[$. This is not compatible with (ii).

Lemma 3 Assume that for some $r \in \mathbb{R}$

$$
v(x, t)>0 \quad \text { if } \quad x \geq r .
$$

In addition $\omega(r) \leq 1$ and there exists a periodic solution $\varphi(t)$ with $\varphi(0)=A$. Then, for any $R>r$ we can find $x_{R}>A$ with $P\left(x_{R}\right)=R$ such that the following statements hold,

$$
\begin{aligned}
& \text { i) }\left[A, x_{R}\right] \subset \mathcal{D} \text {. } \\
& \text { ii) } x(t, \xi) \leq R \text { for each } t \in[0,1], \xi \in\left[A, x_{R}\right] \text {. } \\
& \text { iii) } \max _{\left[A, x_{R}\right]}|h| \geq(R-r) \text { where } h:=i d-P \text {. }
\end{aligned}
$$

Proof. From the previous lemma we know that $] A, \infty[\subset P(\mathcal{D})$ and so there exists $x_{R} \in \mathcal{D}$ such that $P\left(x_{R}\right)=R$. Since $P$ is increasing and $P(A)=A<R$ we conclude that $x_{R}>A$. Since $\mathcal{D}$ is an interval and $A, x_{R} \in \mathcal{D},(i)$ follows.

ii) First we notice that $x_{R}<r$ since $x_{R} \in \mathcal{D}$ but $[r,+\infty[\cap \mathcal{D}=\phi$. By contradiction suppose that $x(\tau, \xi)>R$ for some $\tau \in] 0,1], \xi \in\left[A, x_{R}\right]$. Let $\psi(t)$ be the solution satisfying $\psi(1)=R$ and $\psi(0)=x_{R}$. Let $\left.\left.\tau \in\right] 0,1\right]$ be the first instant such that $\psi(\tau)=r$. We claim that $\tau$ is the only number in $\psi^{-1}(r)$. Indeed, $\psi^{\prime}(\widehat{\tau})=v(\psi(\widehat{\tau}), \widehat{\tau})=v(r, \widehat{\tau})>0$ for any $\widehat{\tau} \in \psi^{-1}(r)$ 
and this forces the uniqueness of $\tau$. The previous discussion implies that $\psi^{\prime}(t)=v(\psi(t), t)>0$ if $t \in[\tau, 1]$. Hence $\psi$ is strictly increasing on $[\tau, 1]$ and $\psi(t) \leq \psi(1)=R$ on this interval. The definition of $\tau$ implies that $\psi(t) \leq r$ on $[0, \tau]$ and so $\psi(t) \leq R$ on $[0,1]$. Finally we observe that, by uniqueness, $x(t, \xi) \leq \psi(t)$ if $t \in[0,1]$ and this is against the assumption $x(t, \xi)>R$ for some $t$.

iii) $x_{R}<r$ since $x_{R} \in \mathcal{D}$ but $r \notin \mathcal{D}$. Since $P$ is increasing, then

$$
\max _{\left[A, x_{R}\right]}|h|=\max _{\left[A, x_{R}\right]}|i d-P| \geq\left|h\left(x_{R}\right)\right|=\left|x_{R}-P\left(x_{R}\right)\right|=\left|x_{R}-R\right|=R-x_{R} \geq R-r .
$$

We conclude this section with an adaptation of Lemma 3 to both sides of the real line. The proof is analogous.

Lemma 4 Assume that for some $r_{+}>0>r_{-}$,

$$
v(x, t)>0 \quad \text { if } \quad x \geq r_{+}, \quad v(x, t)<0 \quad \text { if } \quad x \leq r_{-} .
$$

In addition $\omega\left(r_{+}\right) \leq 1, \omega\left(r_{-}\right) \leq 1$, and there exists at least one periodic solution. Then, for any $R>\max \left\{r_{+},\left|r_{-}\right|\right\}$, we can find an interval $J=[a, b] \subset\left[r_{-}, r_{+}\right]$ such that

i) $J \subset \mathcal{D}, P(J)=[-R, R]$ and all the fixed points of $P$ are contained in $J$.

ii) $|x(t, \xi)| \leq R$ for each $t \in[0,1], \xi \in J$.

iii) $\max _{J}|h| \geq(R-r)$ where $h:=i d-P$.

\section{Estimates for complex-valued solutions}

In this section we consider the complex valued differential equation

$$
z^{\prime}=v(z, t)
$$

where $v: \mathbb{C} \times \mathbb{R} \rightarrow \mathbb{C}$ is continuous, holomorphic in $z$, 1-periodic in $t$ and $v(z, t) \in \mathbb{R}$ if $z \in \mathbb{R}$. Since the time is real, this equation can be interpreted as a system in $\mathbb{R}^{2}$, namely

$$
x^{\prime}=\Re e v(z, t), \quad y^{\prime}=\Im m v(z, t), \quad z=x+i y .
$$

Since the line $y=0$ is invariant we recover the real-valued equation on the $\mathrm{x}$-axis.

We present a result similar to Lemma 2 in [1].

Lemma 5 Assume that we are given a compact interval $[a, b]$ and a rectangle $F=[a-\epsilon, b+\epsilon] \times[-\epsilon, \epsilon]$ for some $\epsilon>0$.

In addition there are positive number $\rho$ and $K$ such that 
- for each $\widehat{\xi} \in[a, b], z(t, \widehat{\xi})$ is well defined in $[0,1]$ and $|z(t, \widehat{\xi})-c| \leq \rho$ for all $t \in[0,1]$, where $c=\frac{a+b}{2}$ is the mid-point of the interval,

- $F$ is contained in the disk of radius $\rho$ centered at $c$ and $|v(z, t)| \leq K$ if $|z-c| \leq \rho+2$

- $\sqrt{2} \epsilon \exp (K)<1$.

Then, for each $\xi \in F$ the solution $z(t, \xi)$ is well defined in $[0,1]$ and satisfies $|z(t, \xi)-c| \leq \rho+1, \quad|z(t, \xi)-\xi| \leq \rho+\frac{b-a}{2}+\sqrt{2} \epsilon(1+\exp (K)), \quad t \in[0,1]$.

Proof. First we notice that from the assumptions $\frac{b-a}{2} \leq \rho$. Given $\xi \in F$ we denote by $\widetilde{\omega}=\widetilde{\omega}(\xi)$ the largest number in $[0,1]$ such that

$$
|z(t, \xi)-c| \leq \rho+1 \quad \text { if } t \in[0, \widetilde{\omega}] .
$$

Given any $\xi$ in $F$ we can find $\widehat{\xi} \in[a, b]$ such that

$$
|\xi-\widehat{\xi}| \leq \sqrt{2} \epsilon .
$$

Then, from the differential equation

$$
|z(t, \xi)-z(t, \widehat{\xi})| \leq|\xi-\widehat{\xi}|+\int_{0}^{t}|v(z(s, \xi), s)-v(z(s, \widehat{\xi}), s)| d s \quad \text { if } \quad t \in[0, \widetilde{\omega}] .
$$

The function $v(., t)$ is holomorphic and Cauchy's inequality implies that for $|z-c| \leq \rho+1$,

$$
\left|\frac{\partial v}{\partial z}(z, t)\right| \leq \max _{|z-c| \leq \rho+2}|v(z, t)| \leq K .
$$

From the complex version of the Mean Value Theorem we deduce that,

$$
|z(t, \xi)-z(t, \widehat{\xi})| \leq|\xi-\widehat{\xi}|+K \int_{0}^{t}|z(s, \xi)-z(s, \widehat{\xi})| d s \quad \text { if } \quad t \in[0, \widetilde{\omega}] .
$$

From Gronwall's lemma,

$$
|z(t, \xi)-z(t, \widehat{\xi})| \leq|\xi-\widehat{\xi}| e^{K t}, t \in[0, \widetilde{\omega}] .
$$

Next we claim that $\widetilde{\omega} \geq 1$. Assuming by contradiction that $\widetilde{\omega}<1$ we notice that $\rho+1=|z(\widetilde{\omega}, \xi)-c| \leq|z(\widetilde{\omega}, \xi)-z(\widetilde{\omega}, \widehat{\xi})|+|z(\widetilde{\omega}, \widehat{\xi})-c| \leq|\xi-\widehat{\xi}| e^{K}+\rho \leq \sqrt{2} \epsilon e^{K}+\rho$, and this is not compatible with the assumptions. In particular the previous reasoning implies that $z(t, \xi)$ is well defined in $[0,1]$ and

$$
|z(t, \xi)-c| \leq \rho+1 \quad \text { if } \quad t \in[0,1] .
$$

To obtain the other estimate we observe that

$$
|z(t, \xi)-\xi| \leq|z(t, \xi)-z(t, \widehat{\xi})|+|z(t, \widehat{\xi})-\widehat{\xi}|+|\widehat{\xi}-\xi| \leq \sqrt{2} \epsilon\left(e^{K}+1\right)+\rho+\frac{b-a}{2}, t \in[a, b] .
$$




\section{An example}

Next we show how to employ the previous results in order to estimate the number of periodic solutions of the equation

$$
x^{\prime}=v(x, t):=x^{3}+\sin x+p(t)
$$

where $p: \mathbb{R} \longrightarrow \mathbb{R}$ is continuous, 1-periodic and $\|p\|_{\infty} \leq C$. The numbers $\pm(C+1)^{\frac{1}{3}}$ are constant lower and upper solutions of this equation and this shows that there exists always at least one periodic solution.(see Theorem 2.1 in $[3])$

Next we define the number

$$
r=2^{\frac{1}{3}}(C+1)^{\frac{1}{3}}
$$

The function

$$
\psi(t)=\frac{r}{\sqrt{1-r^{2} t}}, t \in\left[0, \frac{1}{r^{2}}[\right.
$$

is a solution of $x^{\prime}=\frac{1}{2} x^{3}$ and satisfies $\psi(t) \geq \psi(0)=r$. From these properties it is easy to deduce that $\psi(t)$ is a sub-solution of (11). Actually,

$$
\psi^{\prime}(t)-\psi(t)^{3}-\sin \psi(t)-p(t) \leq-\frac{1}{2} \psi(t)^{3}+1+C \leq-\frac{1}{2} r^{3}+1+C=0 .
$$

In consequence the solution of (11) satisfying $x(0)=r$ must blow up before the time $t=\frac{1}{r^{2}}$. This fact allows up to apply Lemma 4 to (11) with $r_{+}=r, r_{-}=-r$ and $R=2 r$. We find an interval $J=[a, b]$ in the conditions of the Lemma. In particular the number of periodic solutions of the differential equation coincides with the number of zeros of $h=i d-P$ in the interval $[a, b]$.

Also

$$
|x(t, \xi)| \leq 2 r \quad \text { if } \quad t \in[0,1] \quad \text { and } \quad \xi \in J
$$

and

$$
\max _{J}|h| \geq r
$$

Next we apply the results of section 4 . Here it is important to notice that $\sin z$ is an entire function and so

$$
v(z, t)=z^{3}+\sin z+p(t)
$$

is entire as a function of $z$. Let us apply Lemma 5 with

$$
\rho=3 r, \quad K=(4 r+2)^{3}+C+\cosh (\rho+2), \quad \epsilon=\frac{1}{2} e^{-K},
$$

where $\Im z<(\rho+2)$. Let us show that this choice is admissible. Using (12) we notice that if $\widehat{\xi} \in[a, b]$ and $t \in[0,1]$ then

$$
|z(t, \widehat{\xi})-c| \leq|z(t, \widehat{\xi})|+\left|\frac{a+b}{2}\right| \leq 2 r+r=3 r .
$$


Also, since $\epsilon<\frac{1}{2}$, we notice that the rectangle $F$ is contained in the disc centered at $c$ and of radius $3 r$. Moreover, if $|z-c| \leq \rho+2$,

$|v(z, t)| \leq(|z-c|+|c|)^{3}+\cosh (\rho+2)+C \leq(\rho+2+r)^{3}+\cosh (\rho+2)+C:=K$.

From the conclusion of Lemma 5 we know that $h$ is well defined in $F$ and

$$
|h(\xi)| \leq \rho+\frac{b-a}{2}+\sqrt{2} \epsilon\left(1+e^{K}\right) \leq 3 r+r+\frac{\sqrt{2}}{2}\left(e^{-K}+1\right) .
$$

We are ready to apply Theorem 2 to the function $h=i d-P$ on the interval $[a, b]$. Notice that this interval is not symmetric around the origin and we must take into account the Remark after that Theorem. Notice that the theorem on differentiability with respect to initial conditions implies that $h$ is holomorphic. Moreover, since $\Im m z=0$ is invariant for the equation, $h$ is real-valued on the real line. Since $\Gamma=\frac{b-a}{2} \leq r=: \bar{\Gamma}, M=4 r+\frac{\sqrt{2}}{2}\left(e^{-K}+1\right)$ and $m=r$,

$$
N(h,[a, b]) \leq \vartheta \ln \left(4+\frac{\sqrt{2}}{2 r}\left(e^{-K}+1\right)\right)
$$

We can sum up the previous discussions in the following statement:

For each $C>0$ there exists $N(C)$ such that the equation (11) has at most $N$ periodic solutions for any function $p: \mathbb{R} \rightarrow \mathbb{R}$ continuous and 1-periodic with

$$
\|p\|_{\infty} \leq C
$$

Moreover, the number $N$ can be computed numerically.

\section{Acknowledgements}

I thank Prof. R. Ortega for several useful remarks.

\section{References}

[1] Yu Ilyashenko, Hilbert-type numbers for Abel equations, growth and zeros of holomorphic functions, Nonlinearity 13 (2000)1337-1342.

[2] A. I. Markushevich, Theory of Functions of a Complex Variable, Vol II, Prentice-Hall, Englewood Cliffs, NJ, 1965.

[3] M. N. Nkashama, A generalized upper and lower solutions method and multiplicity results for nonlinear first-order ordinary differential equations, J. Math. Anal. Appl. 140 (1989)381-395.

[4] R. Ortega, Counting periodic solutions of the forced pendulum equation, Nonlinear Analysis 42 (2000)1055-1062.

[5] Ch. Pommerenke, Conformal maps at the boundary, Handbook of complex analysis: geometric function theory. Vol 1, pages(39-40) Elsevier 2002. 